\title{
Scalable micro-accordion mesh for deformable and stretchable metallic films
}

\author{
Jan Mertens ${ }^{1}$, Richard W. Bowman ${ }^{1}$, Julian C.W. Willis ${ }^{1}$, Adam Robinson ${ }^{2}$, Darryl Cotton ${ }^{2}$, \\ Richard White $^{2}$, Keith A. Seffen ${ }^{3}$, Jeremy J. Baumberg ${ }^{1 *}$ \\ ${ }^{1}$ NanoPhotonics Centre, Cavendish Laboratory, University of Cambridge, CB3 OHE, UK \\ ${ }^{2}$ Nokia Research Centre, Broers Building, 21 JJ Thomson Avenue, Cambridge CB3 OFA, UK \\ ${ }^{3}$ Department of Engineering, University of Cambridge, Trumpington Street, Cambridge, CB2 1PZ, UK
}

Elastically deformable materials can be created from rigids sheets through patterning appropriate meshes which can locally bend and flex. We demonstrate how micro-accordion patterns can be fabricated across large areas using three-beam interference lithography. Our resulting mesh induces a large and robust elasticity within any rigid material film. Gold coating the micro-accordion produces stretchable conducting films. Conductivity changes are negligible when the sample is stretched reversibly up to $30 \%$ and no major defects are introduced, in comparison to continuous

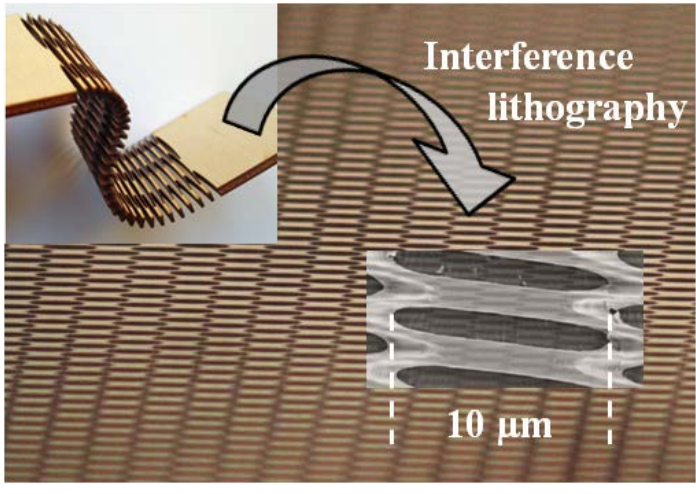
sheets which quickly tear. Scaling analysis shows that our method is suited to further miniaturisation and large-scale fabrication of stretchable functional films. It thus opens routes to stretchable interconnects in electronic, photonic and sensing applications, as well as a wide variety of other deformable structures.

\section{Introduction}

In recent years, the fabrication of elastic conductors has attracted increasing interest. With the development of stretchable materials, novel applications become possible, ranging from stretchable electronics [1-4] through stretchable light emitting diodes (LEDs) [5-8], solar cells [9-11], photodetectors $[12,13]$, and sensors $[14-16]$ to electronic textiles $[17,18]$ and neuronal interfacing for biomedical applications [19-21]. In general, there are two different approaches to fabricate stretchable conductors. One is to use composite soft materials that incorporate conducting additives, for example graphene, carbon nanotubes, metallic nanoparticles and nanowires, or conductive polymers [22-30]. Another possibility is to create stretchability via structural changes of a semi-rigid material using for example controlled buckling of thin metallic films on elastomeric substrates [31-33] or lithographic patterning of metals [34-37]. Lithographically fabricated structures are often composed of individual serpentine wires which for optimal stretch performance need to be structured at the micron-scale while maintaining the capability for volume production. Although suitable lithographic masks can also be created by electron beam lithography with optimised detailed structural features, this is prohibitively slow over large areas, or for producing large area imprint rollers.

Here, we present a novel and scaleable route for the fabrication of robust stretchable structures on the sub- $\mu \mathrm{m}$ scale with feature sizes below $100 \mathrm{~nm}$. Initially stiff and brittle homogeneous photopolymer material is patterned with slots in a periodic fashion using three beam interference lithography. This creates an accordion-like heterogeneous material capable of large and reversible macroscopic strains without introducing global defects or locally compromising brittle strain limits. Furthermore, coating these structures with gold allows for fabrication of elastic conducting films. On a

*jjb12@cam.ac.uk 
macroscale, a similar concept is used for the fabrication of "expanded metal", in which a metal plate is patterned with slits and streched to form a diamond-shaped structure. Applications of this material can be found in many areas of the metal industry and first reports of such metal patterning date back to the $18^{\text {th }}$ century. However, this is, to our knowledge, the first time that a stretchable sub- $\mu$ m mesh is fabricated on a large area using a single-exposure lithography technique. Previous reports of large area stretchable conductors are mainly based on buckling of metallic films or the strain-induced remodelling of granular metallic layers over many cycles [31-33,38], as well as the patterning of elastomeric substrates $[39,40]$. A well defined mesh is, however, of high interest and shows potential for applications [41-43].

(a)

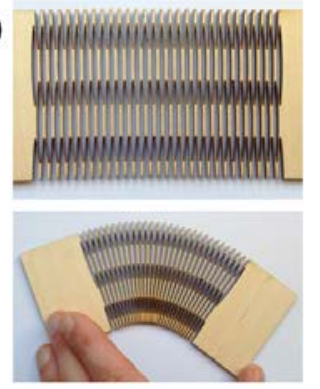

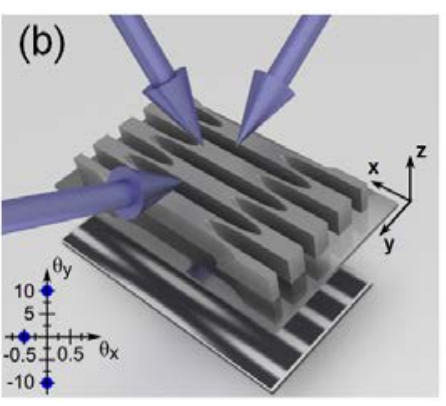

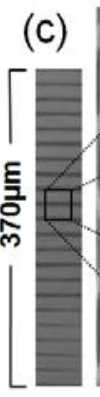

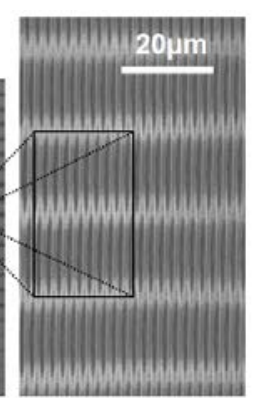

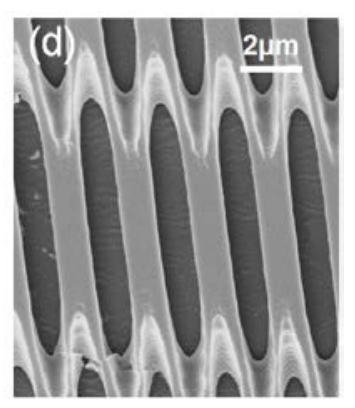

FIG.1. (a) Concept of the accordion spring structure made from $3 \mathrm{~mm}$ thick plywood, showing flexibility. (b) Schematic of interference lithography for fabricating the structure. Inset depicts angles of incident laser beams with respect to surface normal. (c,d) SEM pictures showing (c) the high uniformity and (d) close-up of the structure on a PDMS substrate.

\section{Sample fabrication}

A macro-scale concept model of the accordion spring pattern lasercut into $3 \mathrm{~mm}$-thick plywood shows the essential operation (Fig. 1a). Laser cutting introduces slits in order to produce a heterogeneous periodic slotted structure of thin struts connected to larger bodies. Its mechanical flexibility (bottom image) shows how the structure can be bent, with the accordion springs showing compressibility and stretchability at the same time due to the local bending and twisting of the struts; the interconnecting bodies do not deform but displace rigidly with the strut ends.

On the micrometre scale, samples are fabricated in photoresist using a highly-specific configuration of three beam interference lithography. Three $405 \mathrm{~nm}$ laser beams of equal intensity illuminate a $1.8 \mu \mathrm{m}$ thick photoresist (AZ 5214E) film that is spin coated on a flexible polydimethylsiloxane (PDMS) substrate (Fig. 1b). The inset depicts the required angles of the incident laser beams referenced to the layer normal $(z)$. Reports in the literature study the laser beam arrangement necessary to obtain square or hexagonal lattices in a single-exposure step [45-48]. Instead here, two beams irradiate the photoresist in the $y-z$ plane with an angle of $20^{\circ}$ between them, creating an interference grating in the sample plane. A third beam illuminates the sample in the $x-z$ plane at an angle of $0.5^{\circ}$ to the surface normal, perturbing this interference grating. This perturbation creates an accordion spring structure, seen in the bottom underlay of Fig. 1(b) which shows the calculated interference pattern with white (black) areas as high (low) illumination intensity. By changing the relative angles of grating and perturbing beams, the structures can be scaled down to the deep sub-micron domain and a wide variety of different structures can be generated (Supp. Info. A). For exploring the conductive properties of the mesh, the accordions are evaporatively coated with $40 \mathrm{~nm}$ of $\mathrm{Au}$.

III. Results

A. Mechanical characterisation - Experimental and theoretical 
The accordion structures are uniform over centimetre length scales. A typical scanning electron microscopy (SEM) segment is shown in Fig. 1(c). The white horizontal wavy lines in the SEM image mark the areas of maximum perturbation in the interference grating, seen more clearly in the higher magnification of the positive photoresist structure (Fig. 1d). The structure gains its stretchability from the deep slits. The slope of the wall profile at the connecting struts is set by the absorption of the photoresist while the aspect ratio of the slits (slit length $L$ to width $W$, Fig. $2 \mathrm{e}$ ) is defined by the absorption of the resist, the resist thickness, and the angle between the perturbing laser beam and the surface normal (Supp. Info. A) [44]. All these can be controlled by varying the resist parameters, and for the situation here the slits have an aspect ratio of $\sim 10$. This aspect ratio can be further increased by using thinner photoresist or illuminating for longer, but is limited as the structure becomes more fragile leading to larger defect areas (Supp. Info. B [44]). Struts in the interpenetration area are thin at the top (width $\sim 100 \mathrm{~nm}$ ) and widen towards the PDMS substrate following the absorption profile of the photoresist.

(a)

(b)
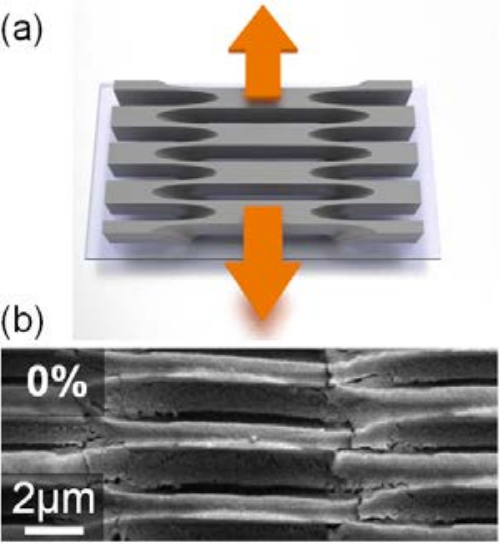

(c)

(d)
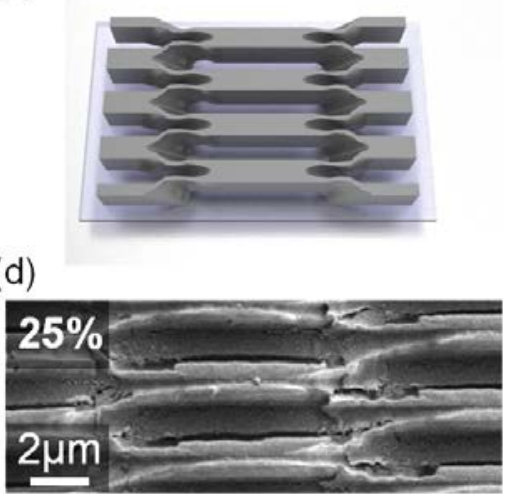

(e)

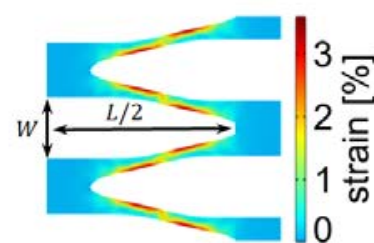

(f)

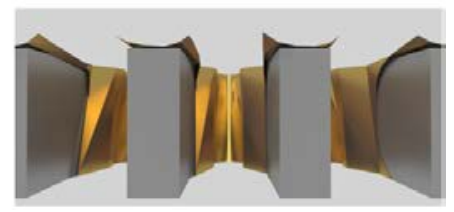

FIG.2. (a-d) Accordion spring structure in relaxed (left col.) and stretched state (right col.), shown in schematic $(a, c)$ and SEM (b,d), orange arrows depict stretch direction. (e) Simulation of the local strain at the top surface when the full structure is extended by $20 \%$. (f) Schematic cross section of stretched structure illustrating the Au butterfly effect when stretched.

The accordion spring structures are uniaxially stretched perpendicular to the narrow slits (Fig.2a orange arrows) with a computer-controlled stage. An SEM image (taken at $30^{\circ}$ viewing) of the relaxed Au-coated sample after repeated stretching cycles is shown in Fig. 2(b). Small cracks in the Au coating are visible at the thin connecting struts which only emerge after prolonged stretch cycling. Stretching leads to bending of the relatively straight struts into gentle S-curves and widening of the slits between the interconnected bodies, producing a macroscopic strain of the accordion structure (Fig. 2c,d). Twisting of struts occurs along two axes, in-plane deformations initially and then out of plane as discussed below. To gain insight into the degree of local strain within the struts, a finite element simulation of the mechanical properties of the full structure, i.e. of the patterned photoresist including the PDMS substrate, is performed (Supp. Info. D [44]). Here, however, we focus on the simulation results of the strain at the top surface since cracks in gold coatings follow this strain distribution. A discussion of strain in and at the bottom of the structure is given in the supplementary material. When the accordion spring structure is for example stretched by $20 \%$, the local strains are highly concentrated along the connecting struts (Fig. 2e), where the maximum material strain is approximately $3 \%$. Even a strain of $3 \%$ in the experiment would likely lead to an electrical failure of the device, which is not observed. Therefore, additional stress reduction processes take place which are not included in the model. One of these processes is the planar deformation of the struts that induces crack formation of the overlying gold coating seen in the SEMs. The strain diffuses rapidly into the main bodies which are not strained across their bulk, accounting for the robustness and 
elastic stretching of the design. As well as deforming in-plane, the struts undergo orthogonal straining in the out-of-plane direction, resulting in a surface displacement. These strains follow from the rigid boundary conditions between the overall structure and the underlying PDMS substrate, which is confirmed by finite element simulations (Supp. Info. D) [44]. This indicates that the surface displacement causes the Au coating to delaminate selectively away from the sidewalls of the main ribs, which can be seen directly in the centre of the spring structure (Fig. 2d). The delamination is visible as sub-micron $\mathrm{Au}$ wings that bend upwards when the sample is stretched, illustrated schematically in cross section in Fig. 2(f). This Au film buckling is reversible upon relaxing the strain, giving rise to three dimensional dynamical reconfiguration (like a butterfly wing) induced from the initial 2D patterning, while no damage is introduced to the photoresist ribbons directly underneath. The Au wing delamination is an additional process that reduces stress in the Au layer to keep the film intact. No Au lift-off from the ribbons is observed when stretching the mesh which suggests good structural strain relief and strong adhesion between gold film and photoresist.

\section{B. Optical characterisation}

The planar deformation is further studied by optical characterisation of the Au-coated micro-accordion sheets. Samples are mounted in a computer-controlled translation stage inside a microscope, enabling optical micrographs to be recorded while stretching the samples horizontally (Fig. 3a). Darker regions show the slits while the bright golden areas correspond to the Au-coated photoresist. Quantitative characterisation of these flexible diffraction gratings uses blue laser light incident through the microscope onto the sample giving diffracted light observed in the back focal plane of the microscope objective using a Bertrand lens. The diffraction pattern of white light in transmission is shown in the inset, showing the first diffraction orders and allowing calibration of the structure periodicity via the diffraction equation. The red arrows indicate a span of 10 accordion springs revealing the slits opening up as a strain of $10 \%$ is applied. The structure responds linearly with a $10 \%$ increase in the periodicity confirmed by the diffraction measurements (Fig. 3b). The linearly increasing periodicity with strain confirms that the patterned photoresist remains fully attached to the underlying PDMS substrate without delamination.

(a)

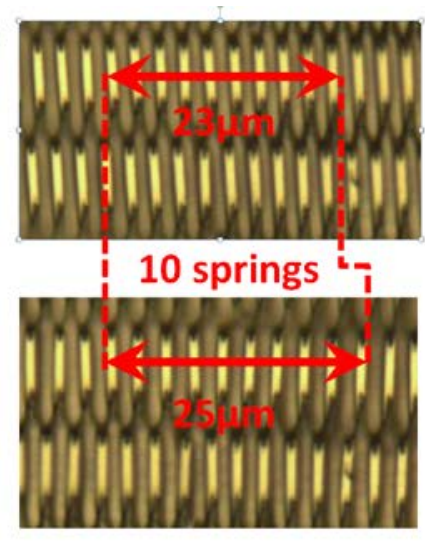

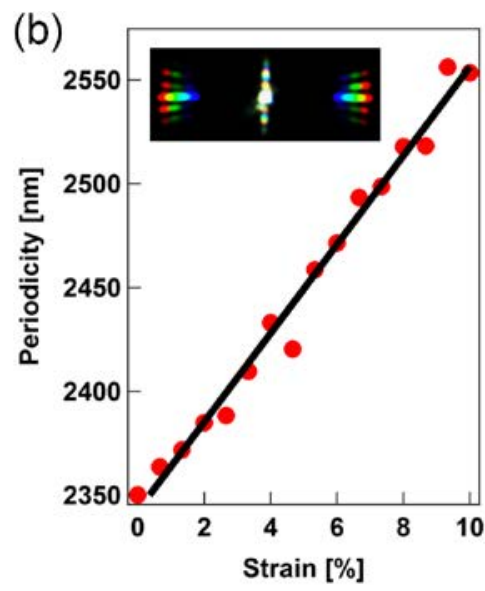

FIG.3. (a) Microscope images of micro-accordion in relaxed (top) and stretched (bottom) state. Red arrows mark width of 10 accordion springs showing $10 \%$ stretch. (b) Corresponding periodicity measured from white-light diffraction (inset) while stretching.

\section{Electronic characterisation}


The Au-coated micro-accordions are contacted with two micromanipulator probes for conductive measurements to reveal how such accordion spring films can be used as elastic conductors. Optical images of a contacted sample in relaxed and stretched states are shown in insets of Fig. 4(a). In each step, the probes are lifted up before the sample is stretched and afterwards placed down on the same rib to ensure conductivity measurements over a fixed number of accordion springs. Samples stretched up to $30 \%$ show that the resistance is almost constant over the entire range. Fluctuations in the resistance are mainly due to variations in contact resistance with repeated probe tip landings on the same ribs. While four-point electrical measurements are currently not feasible in such dense meshes, these results still demonstrate the lack of any strong increase in sheet resistance. While stretching of the samples beyond $30 \%$ results in rupture of the PDMS substrate, no failure of the Aucoated accordion pattern is observed. Small micro-cracks formed when stretching the sample or local defects from the lithography process do not significantly influence the conductivity. Stretching and relaxing of samples is fully reversible and even when cycled more than 100 times no failure of the device is discerned. Results of a durability test are shown in Fig. 4(b). A sample is stretched by $30 \%$ in each cycle and the resistance is measured in the stretched state. For the first 20 stretch cycles, an unexpected fast drop in resistance is observed. This decrease in resistance continues to develop over the next 80 cycles showing that the micro-accordion sheet does not lose its conductivity but improves with cycling. The drop in resistance is likely explained by the formation of complex networks of microcracks in the stretched and bent metal coatings. Such crack networks are observed in the SEM images of Fig. 2(b,d) and widely reported in the literature [32]. Cracks reduce in size over many cycles, allowing the metal to better wrap around strut edges and thus reducing the resistance of the linker struts which form local bottlenecks for electrical transport. Furthermore, such cracks reduce the residual strain in the gold coating of the mesh pattern, which can additionally lead to a reduction of the Au resistance after multiple stretch cycles.
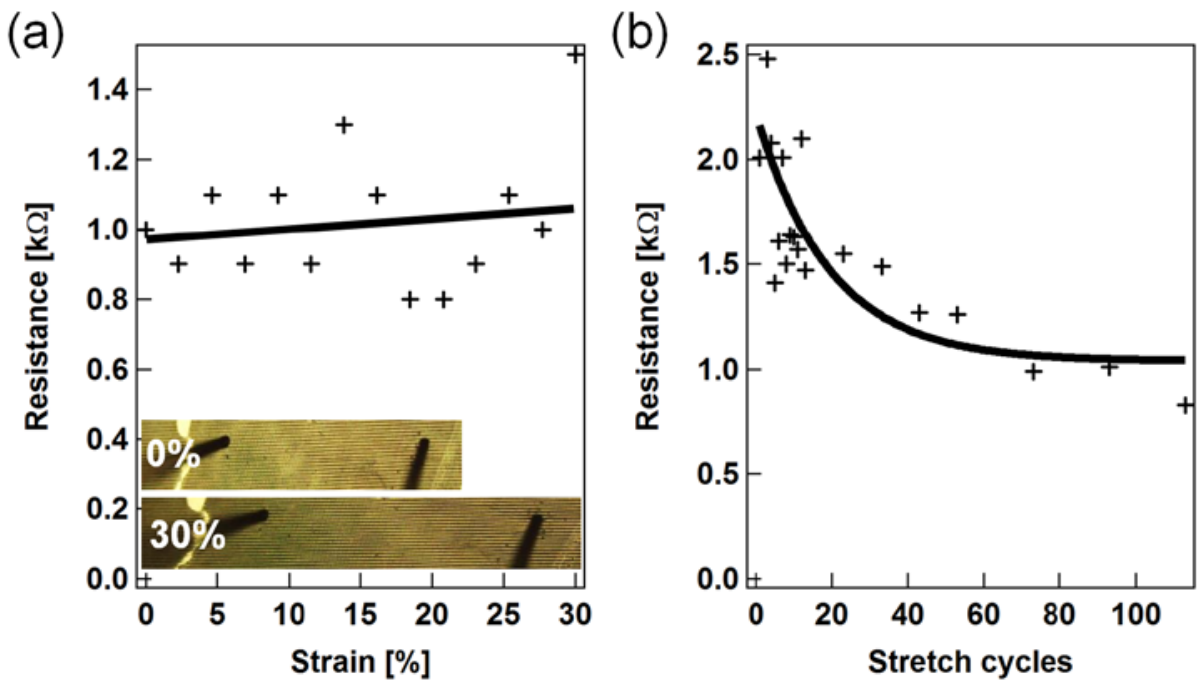

FIG.4. (a) Resistance measurement of micro-accordion sheet vs strain. Insets show in-situ microprobes for conducting measurements on the accordion pattern in relaxed (top) and stretched (bottom) states. (b) Resistance vs stretch cycles of the Au coated sheet.

\section{Discussion}

The advantage of an interference lithographic route to such deformable micro-accordion networks is the strong prospect for scalable films, using it either directly, or by exploiting it to create masters for embossing in a roll-to-roll continuous process. Structure sizes down below $100 \mathrm{~nm}$ are possible simply by moving to deeper UV laser processing. A simplified analytical model of the accordion mesh shows that reducing the strut width and increasing the aspect ratio of slits results in a reduction of the 
force required for elastic deformations. In this model, only deformations of the connecting struts are assumed, following our observations above. Out-of-plane deformations of the mesh along the surface normal are neglected.
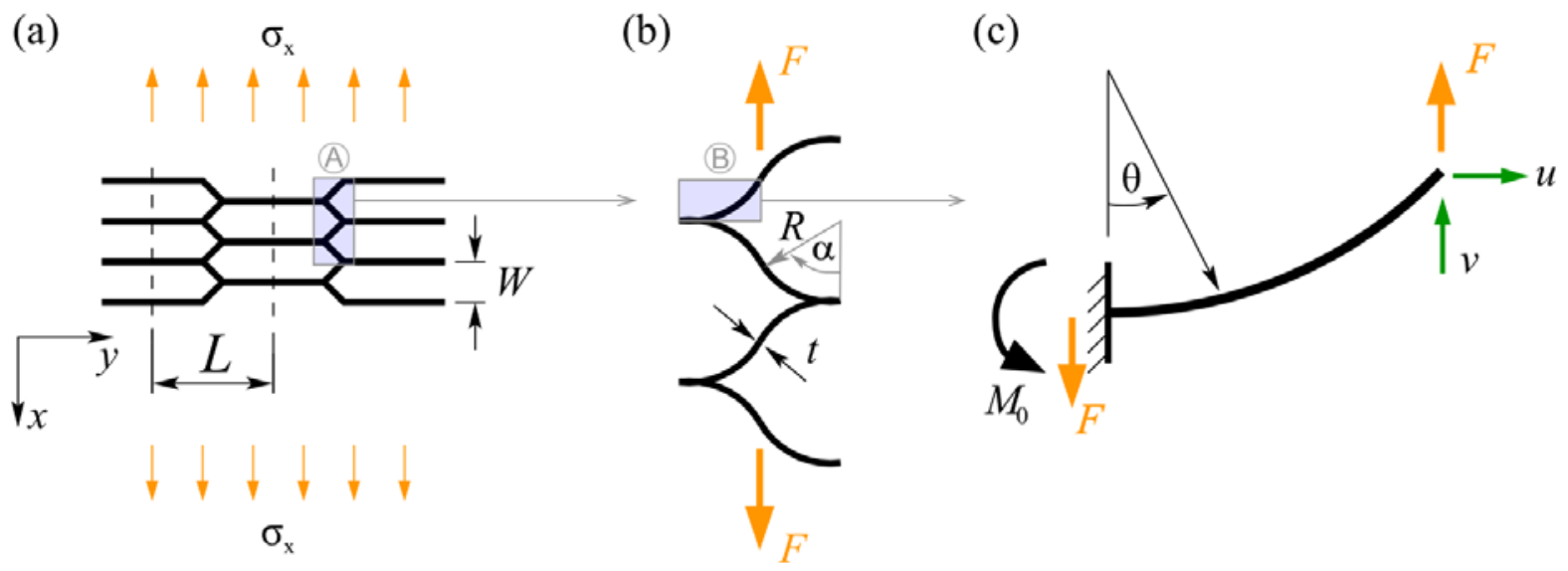

FIG.5. Analytical model for estimating tensile performance of the accordion mesh. (a) Schematic layout of the mesh under uniaxial long-range stress $\sigma_{x}$. Dimensions of a typical unit cell are $W \times L$. (b) Enlarged blue box A showing the junction line of in-plane thickness $t$, acted on by opposing forces $F$ which arise from $\sigma_{x}$. Stress free radius of curvature is $R$ subtending $\alpha$. (c) One-half of a typical junction beam, box $\mathrm{B}$, behaving as a tip-loaded cantilever embedded at the opposite end. The corresponding displacements under $F$ are $u$ and $v$, and $M_{0}$ is the moment reaction transferred at the connection point, along with $F$, to the next junction beam (not shown).

The considered simplified mesh is sketched in Fig. 5(a). It is stretched along the $x$-direction due to application of a uniaxial long-range stress $\sigma_{x}$. Deformation of the mesh is localised in the junction region (blue box A). Single junction beams (Fig. 5b) are defined by a pair of equal but oppositely curved cantilevers (blue box $\mathrm{B}$ ) with an initial stress-free radius of curvature $R$, subtending angle $\alpha$. The forces acting on one half of a junction beam are displayed in Fig. 5(c). This force induces an inplane displacement $u$ and $v$ as well as a bending moment $M_{0}$, from the connection to the next junction beam. Deformations of the beams can be well approximated by a tip-loaded curved cantilever. The tip of this cantilever is displaced under the applied load, describing axial strains $\varepsilon_{x}, \varepsilon_{y}$ in the unit cell:

$$
\varepsilon_{x}=\frac{4 v}{L}, \quad \varepsilon_{y}=\frac{4 u}{W} .
$$

Here, a factor 4 is included because each unit cell consists of 4 cantilever beams in the junction line. The tip displacement $\boldsymbol{\delta}=(v, u)$ of the cantilever is obtained following the method of virtual work for beams and frames (see Supp. Info. C) [44]:

$$
P^{*} \delta=\int M^{*} \kappa d s,
$$

where $P^{\star}$ is the applied 'virtual' load at the point of interest in the desired direction, $M^{\star}$ is the corresponding virtual bending moment, $\kappa$ is the change in beam curvature of when a load is applied, and $d s$ is the beam arc-length. The bending moment varies along the cantilever and is hence a function of the angle $\theta$, of the applied force $F$, and of the radius of curvature $R$ of the stress free cantilever (Fig. 5c):

$$
M(\theta)=F(R \sin \alpha-R \sin \theta) .
$$

The change in curvature depends on the Young's modulus $E$ of the material and the second area moment of the cross section about the bending axis $I=b t^{3} / 12$ with the cantilever depth $b$. It is obtained from the constitutive elastic law for slender beams, 


$$
\kappa=M(\theta) / E I .
$$

Inserting equations (3) and (4) in equation (1) and integrating with an applied virtual unit force $P^{\star}=1^{\star}$ provides the beam displacements along the $x$ - and $y$-directions (Supp. Info C) [44]. Since $F$ depends on $\sigma_{x}$, an effective axial modulus for the mesh can then be defined,

$$
\frac{\sigma_{x}}{\varepsilon_{x}} \propto E\left(\frac{t}{R}\right)^{3} \frac{W}{L} .
$$

This effective axial modulus in Eq.(5) is inversely proportionally to the aspect ratio $L / W$, as well as scaling with the cube of strut thickness to the link strut curvature. Increasing the aspect ratio thus linearly decreases the force needed to elastically stretch the mesh. The aspect ratio can be directly controlled via the precise angular laser beam arrangement, as shown in the supplementary material (Supp. Info. A) [44]. Additionally exposure and development allow control of strut thickness. This analysis shows how further reduction of feature sizes will ensure that the required operational forces can be also reduced.

As well as films on flexible substrates, free standing films can be floated off. However, free standing films are difficult to handle and are therefore often not suitable for applications. By combining accordion-spring motifs in different orientations on the resist films, for instance using a spatial light modulator to direct different beams to different regions of the film, omnidirectional accordion stretching can be produced (Supp. Info. E) [44]. Similarly although we demonstrate only up to $30 \%$ strain so far, limited by our choice of substrate material, larger strains can be supported by the accordion springs, with limits still to be fully understood. While we use here simple Au-coating of the patterned photoresist, the material choice can be widely extended to many metallic and multilayer structures using lift-off, moulding, or printing techniques (Supp. Info. B) [44]. As a result, this opens up a large variety of nanopatterned stretchable film designs that can have optical, electronic, magnetic and sensing functionality built-in, yet be low cost and large area.

For example, complicated structures can be generated when multiple exposures are used. The calculated interference patterns of double exposures with 3 beams are presented in Fig. 6 . In both cases, all three laser beams have an angle $\theta=10^{\circ}$ with respect to the surface normal. In (a), the sample is rotated by $\alpha=10^{\circ}$ after the first exposure, while a sample rotation of $\alpha=30^{\circ}$ is used in (b). Both configurations generate porous resist films that allow for a controlled fabrication route to stretchable, sponge-like thin films. Stretching structure (a) leads to a displacement of the repeating units accompanied by rotations of connections as indicated, so that conductive contacts remain intact.

(a)
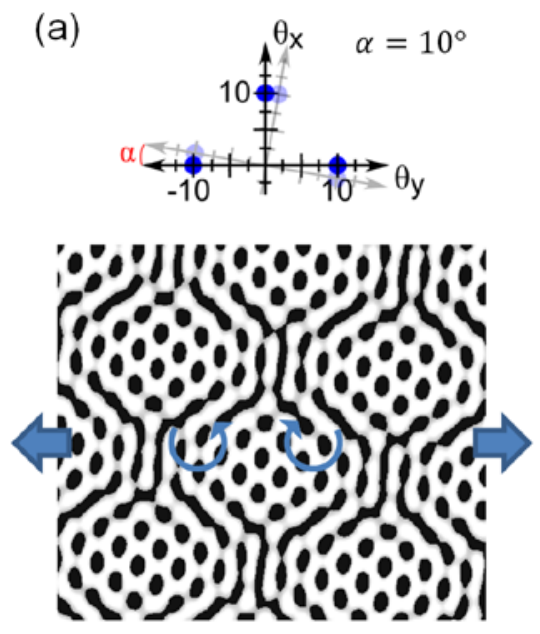

(b)
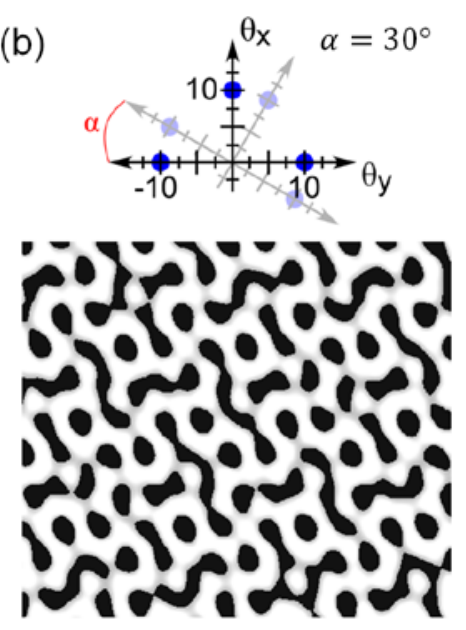

FIG. 6. Calculated interference patterns of more complex structures. A double exposure is used in which the sample is rotated after the first exposure step by (a) $\alpha=10^{\circ}$, and (b) $\alpha=30^{\circ}$ as indicated in the diagrams at the top. 
However, scaling up of such fabrication processes to large areas is more complicated than for the accordion spring pattern due to the additional rotation step of the substrate relative to the illuminating beams. Therefore, the accordion mesh is chosen here for fabrication with single-exposure 3-beam interference lithography. In addition we note that electron beam lithography can access additional geometries of such nanostructures for micro-scale testing, but as discussed above, the fabrication process cannot be applied on the large scales needed for a large-area process. Further work is thus focussed on optimising pattern geometries that are both accessible, have high yield, and can be scaled to large areas.

\section{Conclusion}

In conclusion, we have presented a novel approach to fabricating stretchable microstructures by patterning photoresist using three-beam interference lithography in a specific orientation. Key to this approach is creating long cuts into a brittle material that can then respond through strut bending and twisting. The resulting accordion spring patterns are robust, and with strongly-interpenetrating slits they can be highly stretched. Elasticity measurements show the film's potential as its conductivity is maintained on stretching. Micro-accordion patterning thus generates a new route for the fabrication of elastic metals. Further miniaturisation and functionalization of the structure can be achieved by changing the illuminating geometry in the lithography, and adapting to incorporate new materials. Our analysis shows that the elastic properties improve as these patterns are miniaturised, giving strong motivation for such nanostructuring. Our approach to use interference lithography enables large-scale fabrication of lithographically defined stretchable metallic films and thus opens up new routes to stretchable interconnects and pads in electronics, photonics and sensing applications.

\section{Acknowledgements:}

We are grateful for funding from the Cambridge NanoDTC, ERC LINASS 320503 and UK EPSRC grants EP/G060649/1, EP/G037221/1, EP/L027151/1 and EP/L015978/1, as well as Nokia Research. RWB thanks Queens' College Cambridge for financial support. JM acknowledges support from the Winton Programme for the Physics of Sustainability.

\section{Appendix A: Experimental details}

\section{A. Fabrication of PDMS substrates:}

PDMS (Sylgard 184 Elastomer Kit, Dow Corning) was prepared using a 10:1 degassed mix of base and curing agent that was cast in glass dishes with cleaned $1.5 \times 1.5 \mathrm{~cm}^{2}$ glass substrates. These substrates were covered by $1 \mathrm{~mm}$ of PDMS and baked in an oven for $4 \mathrm{~h}$ at $70^{\circ} \mathrm{C}$. The cured PDMS was cut free from the glass dish with a $3 \mathrm{~mm}$ border around the glass substrates, which were subsequently removed. The support border was glued on a larger glass substrate to allow simple handling without contact between glass and PDMS.

\section{B. Interference lithography:}

The PDMS substrates were cleaned with acetone and IPA before photoresist (AZ 5214E) was spin coated on the dried substrates with $8000 \mathrm{rpm}$ for $30 \mathrm{~s}$ to gain a film with a thickness of $1.8 \mu \mathrm{m}$. A soft baking step with $115^{\circ} \mathrm{C}$ for $60 \mathrm{~s}$ ensured that remaining solvent was removed. Illumination of a circular area with a diameter of $1.4 \mathrm{~cm}$ was performed with three $405 \mathrm{~nm}$ laser beams and a total intensity of 
$12 \mathrm{~mW}$ for $14 \mathrm{~s}$. The sample remained in a developing bath with a 1:4 solution of AZ 351B developer and deionised water for $50 \mathrm{~s}$. Electron beam evaporation was used to coat the developed samples with $40 \mathrm{~nm}$ of $\mathrm{Au}$.

\section{Stretch measurements:}

Stretch measurements were performed on a computer controlled linear translation stage mounted in a microscope. For the optical characterisation, an adapted Olympus BX51 microscope in bright field configuration was used. The structure morphology was examined in a scanning electron microscope (SEM) equipped with an extensometer.

\section{Electric measurements:}

Resistance measurements were performed by contacting the samples with two microprobes (20 $\mu \mathrm{m}$ gold wires) attached to micromanipulators (Kleindiek Nanotechnik) to ensure precise positioning of the probes. A voltage of $150 \mu \mathrm{V}$ (Keithley $2635 \mathrm{~A}$ ) was applied to the sample and the resistance was measured.

\section{E. Diffraction measurements:}

White light from an incandescent source or a laser with wavelength of $448 \mathrm{~nm}$ (Coherent Cube) illuminated the sample with parallel light rays through the microscope objective. Diffracted light was collected and imaged in the back focal plane of the objective using a Bertrand lens.

\section{F. Simulations:}

Strain simulations were performed using commercial software (COMSOL). The photoresist AZ 5124E was modelled as a linear elastic material with a Young's Modulus of 7 GPa [49]; PDMS was modelled as a hyperelastic material using the Mooney-Rivlin fit with parameters $E=1.23 \mathrm{MPa}, \mathrm{C} 1=0.205$ $\mathrm{MPa}, \mathrm{C} 2=0 \mathrm{MPa}$ (corresponding to a 24 hour cure at $60{ }^{\circ} \mathrm{C}$ ) [50]. Symmetric boundary conditions were applied at each vertical face. A displacement boundary condition was applied to each end of the PDMS/AZ structure in order to provide a total applied strain of $20 \%$. The simulation included nonlinear geometrical effects. Post-processing strain slices were recorded at the base and the surface of the AZ structure. 


\section{References:}

[1] M. Park, J. Im, M. Shin, Y. Min, J. Park, H. Cho, S. Park, M.-B. Shim, S. Jeon, D.-Y. Chung, J. Bae, J. Park, U. Jeong, and K. Kim, Nat. Nanotechnol. 7, 803 (2012).

[2] M. Kaltenbrunner, T. Sekitani, J. Reeder, T. Yokota, K. Kuribara, T. Tokuhara, M. Drack, R. Schwödiauer, I. Graz, S. Bauer-Gogonea, S. Bauer, and T. Someya, Nature 499, 458 (2013).

[3] A. Chortos, J. Lim, J. W. F. To, M. Vosgueritchian, T. J. Dusseault, T.-H. Kim, S. Hwang, and Z. Bao, Adv. Mater. 26, 4253 (2014).

[4] Y.-C. Lai, Y.-C. Huang, T.-Y. Lin, Y.-X. Wang, C.-Y. Chang, Y. Li, T.-Y. Lin, B.-W. Ye, Y.-P. Hsieh, W.-F. Su, Y.-J. Yang, and Y.-F. Chen, NPG Asia Mater. 6, e87 (2014).

[5] T. Sekitani, H. Nakajima, H. Maeda, T. Fukushima, T. Aida, K. Hata, and T. Someya, Nat. Mater. 8, 494 (2009).

[6] R.-H. Kim, D.-H. Kim, J. Xiao, B. H. Kim, S.-I. Park, B. Panilaitis, R. Ghaffari, J. Yao, M. Li, Z. Liu, V. Malyarchuk, D. G. Kim, A.-P. Le, R. G. Nuzzo, D. L. Kaplan, F. G. Omenetto, Y. Huang, Z. Kang, and J. a Rogers, Nat. Mater. 9, 929 (2010).

[7] Z. Yu, X. Niu, Z. Liu, and Q. Pei, Adv. Mater. 23, 3989 (2011).

[8] H. L. Filiatrault, G. C. Porteous, R. S. Carmichael, G. J. E. Davidson, and T. B. Carmichael, Adv. Mater. 24, 2673 (2012).

[9] J. Lee, J. Wu, M. Shi, J. Yoon, S.-I. Park, M. Li, Z. Liu, Y. Huang, and J. a Rogers, Adv. Mater. 23, 986 (2011).

[10] D. J. Lipomi, B. C.-K. Tee, M. Vosgueritchian, and Z. Bao, Adv. Mater. 23, 1771 (2011).

[11] M. Kaltenbrunner, M. S. White, E. D. Głowacki, T. Sekitani, T. Someya, N. S. Sariciftci, and S. Bauer, Nat. Commun. 3, 770 (2012).

[12] P. Bartu, R. Koeppe, N. Arnold, A. Neulinger, L. Fallon, and S. Bauer, J. Appl. Phys. 107, 123101 (2010).

[13] Y. M. Song, Y. Xie, V. Malyarchuk, J. Xiao, I. Jung, K.-J. Choi, Z. Liu, H. Park, C. Lu, R.-H. Kim, R. Li, K. B. Crozier, Y. Huang, and J. a Rogers, Nature 497, 95 (2013).

[14] T. Someya, Y. Kato, T. Sekitani, S. Iba, Y. Noguchi, Y. Murase, H. Kawaguchi, and T. Sakurai, Proc. Natl. Acad. Sci. U. S. A. 102, 12321 (2005).

[15] D. J. Lipomi, M. Vosgueritchian, B. C.-K. Tee, S. L. Hellstrom, J. a Lee, C. H. Fox, and Z. Bao, Nat. Nanotechnol. 6, 788 (2011).

[16] M. Ying, A. P. Bonifas, N. Lu, Y. Su, R. Li, H. Cheng, A. Ameen, Y. Huang, and J. a Rogers, Nanotechnology 23, 344004 (2012).

[17] C. Yan, W. Kang, J. Wang, M. Cui, X. Wang, C. Y. Foo, K. J. Chee, and P. S. Lee, ACS Nano 8, 316 (2014).

[18] S. Yao and Y. Zhu, Nanoscale 6, 2345 (2014). 
[19] K. Takei, T. Takahashi, J. C. Ho, H. Ko, A. G. Gillies, P. W. Leu, R. S. Fearing, and A. Javey, Nat. Mater. 9, 821 (2010).

[20] C. M. L. Bozhi Tian, Jia Liu, Tal Dvir, Lihua Jin, Jonathan H. Tsui, Quan Qing Zhigang Suo, Robert Langer, Daniel S. Kohane, Nat. Mater. 11, 872 (2012).

[21] Liang Guo, G. S. Guvanasen, Xi Liu, C. Tuthill, T. R. Nichols, and S. P. DeWeerth, IEEE Trans. Biomed. Circuits Syst. 7, 1 (2013).

[22] K.-Y. Chun, Y. Oh, J. Rho, J.-H. Ahn, Y.-J. Kim, H. R. Choi, and S. Baik, Nat. Nanotechnol. 5, 853 (2010).

[23] Y. Kim, J. Zhu, B. Yeom, M. Di Prima, X. Su, J.-G. Kim, S. J. Yoo, C. Uher, and N. a Kotov, Nature 500, 59 (2013).

[24] Z. Xu, Z. Liu, H. Sun, and C. Gao, Adv. Mater. 25, 3249 (2013).

[25] C. Yan, J. Wang, X. Wang, W. Kang, M. Cui, C. Y. Foo, and P. S. Lee, Adv. Mater. 26, 943 (2014).

[26] M. Park, J. Park, and U. Jeong, Nano Today 9, 244 (2014).

[27] Y. Yu, J. Zeng, C. Chen, Z. Xie, R. Guo, Z. Liu, X. Zhou, Y. Yang, and Z. Zheng, Adv. Mater. 26, 810 (2014).

[28] M. Bernardi, M. Palummo, and J. C. Grossman, Nano Lett. 13, 3664 (2013).

[29] Y. Cheng, S. Wang, R. Wang, J. Sun, and L. Gao, J. Mater. Chem. C 2, 5309 (2014).

[30] S. Savagatrup and A. Printz, Chem. Mater. 26, 3028 (2014).

[31] S. P. Lacour, J. Jones, and S. Wagner, Proc. IEEE 93, 1459 (2005).

[32] S. P. Lacour, D. Chan, S. Wagner, T. Li, and Z. Suo, Appl. Phys. Lett. 88, 204103 (2006).

[33] D.-Y. Khang, J. a. Rogers, and H. H. Lee, Adv. Funct. Mater. 19, 1526 (2009).

[34] Y. Zhang, S. Xu, H. Fu, J. Lee, J. Su, K.-C. Hwang, J. a. Rogers, and Y. Huang, Soft Matter 9, 8062 (2013).

[35] Y. Zhang, S. Wang, X. Li, J. a. Fan, S. Xu, Y. M. Song, K.-J. Choi, W.-H. Yeo, W. Lee, S. N. Nazaar, B. Lu, L. Yin, K.-C. Hwang, J. a. Rogers, and Y. Huang, Adv. Funct. Mater. 24, 2028 (2014).

[36] S. Xu, Y. Zhang, J. Cho, J. Lee, X. Huang, L. Jia, J. a Fan, Y. Su, J. Su, H. Zhang, H. Cheng, B. Lu, C. Yu, C. Chuang, T.-I. Kim, T. Song, K. Shigeta, S. Kang, C. Dagdeviren, I. Petrov, P. V Braun, Y. Huang, U. Paik, and J. a Rogers, Nat. Commun. 4, 1543 (2013).

[37] J. A. Fan, W.-H. Yeo, Y. Su, Y. Hattori, W. Lee, S.-Y. Jung, Y. Zhang, Z. Liu, H. Cheng, L. Falgout, M. Bajema, T. Coleman, D. Gregoire, R. J. Larsen, Y. Huang, and J. a Rogers, Nat. Commun. 5, 3266 (2014).

[38] J. A. Rogers, T. Someya, and Y. Huang, Science 327, 1603 (2010).

[39] P. Mandlik, S. Lacour, and J. Li, Electron Device Lett. IEEE 27, 650 (2006). 
[40] T. Adrega and S. P. Lacour, J. Micromechanics Microengineering 20, 055025 (2010).

[41] T. Sekitani and T. Someya, Adv. Mater. 22, 2228 (2010).

[42] T. Takahashi, K. Takei, A. G. Gillies, R. S. Fearing, and A. Javey, Nano Lett. 11, 5408 (2011).

[43] T. Yamada, Y. Hayamizu, Y. Yamamoto, Y. Yomogida, A. Izadi-Najafabadi, D. N. Futaba, and K. Hata, Nat. Nanotechnol. 6, 296 (2011).

[44] See Supplemental Material at ... for additional theoretical and experimental information.

[45] J. De Boor, N. Geyer, U. Gösele, and V. Schmidt, Opt. Lett. 34, 1783 (2009).

[46] J. L. Stay, G. M. Burrow, and T. K. Gaylord, Rev. Sci. Instrum. 82, 023115 (2011).

[47] H. Hauser, B. Michl, S. Schwarzkopf, V. Kuebler, C. Mueller, M. Hermle, and B. Blaesi, IEEE J. Photovoltaics 2, 114 (2012).

[48] T.-H. Lin, T.-B. Huang, Y.-K. Yang, K.-C. Tseng, and C.-C. Fu, Jpn. J. Appl. Phys. 53, (2014).

[49] C. E. Foerster, I. T. S. Garcia, F. C. Zawislak, F. C. Serbena, C. M. Lepienski, W. H. Schreiner, and M. Abbate, Thin Solid Films 411, 256 (2002).

[50] D. P. J. Cotton, a. Popel, I. M. Graz, and S. P. Lacour, J. Appl. Phys. 109, 054905 (2011). 